\title{
Programa de Aprimoramento e Revitalização do Conhecimento na Enfermagem e Saúde
}

Luiz Anildo Anacleto da Silva(a)

Rafael Marcelo Soder ${ }^{(b)}$

Cínthia Cristina Oliveski(c)

Mirian Natali Frizzo(d)

Silva LAA, Soder RM, Oliveski CC, Frizzo MN. Program for Improvement and Revitalization of Knowledge in Nursing and Health. Interface (Botucatu). 2018; 22(65):517-26.

This paper aimed to understand how meaningful learning can contribute to the construction of knowledge and education in the perspective of Nursing students. This is a qualitative, descriptive and exploratory research involving 12 college students from a Nursing course who were in a teaching program. The results enabled the definition of two categories: contributions in Nursing education and knowledge construction. It follows that offering educational programs with alternative methodologies contributes to the construction of knowledge, education and Nursing. One of the main contributing factors is the possibility of collective edification, making the students to have a critical, reflexive and active participation in the educational activities.

Keywords: Nursing. Nursing education. Nursing students.
Objetivo deste trabalho foi compreender como a aprendizagem significativa pode contribuir para a construção do conhecimento e formação na perspectiva de estudantes de Enfermagem. Trata-se de uma pesquisa qualitativa, descritiva e exploratória. Os sujeitos da pesquisa foram 12 estudantes de um curso de Enfermagem participantes de um programa de ensino. Os resultados permitiram a construção de duas categorias: contribuições na formação em Enfermagem e na construção do conhecimento. Conclui-se que o oferecimento de programas educativos com metodologias alternativas contribui para a construção do conhecimento, formação e Enfermagem. Um dos principais fatores contributivos refere-se à possibilidade da construção coletiva, proporcionando aos estudantes uma participação ativa, crítica e reflexiva nas atividades educativas.

Palavras-chave: Enfermagem. Educação em Enfermagem. Estudantes de Enfermagem. (a, b, c, d) Curso de Enfermagem, Departamento de Ciências da Saúde, Universidade Federal de Santa Maria. Campus de Palmeira das Missões, Av. Independência, 3751 , Bairro Vista Alegre. Palmeira das Missões, RS, Brasil. 98300-000 luiz.anildo@ yahoo.com. br; rafaelsoder@ hotmail.com; cinthia.oliveski@ yahoo.com.br; miriannatali@ hotmail.com 


\section{Introdução}

A interface que compreende a celeridade na produção de conhecimentos na saúde, as transformações sociais, políticas e econômicas, no entrecorte com a rigidez dos planos políticos pedagógicos nas instituições de ensino, associados à compartimentação das áreas do conhecimento, à formatação das disciplinas, à forma de condução dos conteúdos, à verticalização do ensino, motiva, desafia e induz os docentes a conceberem, a preverem e proverem formas complementares de ensino/aprendizagem. Ambientes, espaços e contextos vividos nos desafiam a repensar estratégias pedagógicas alternativas, sobretudo, em agregar estratégias educativas que proporcionem, aos discentes, a participação e autonomia no processo de aprendizagem e construção do conhecimento.

O nominado Programa de Aprimoramento e Revitalização do Conhecimento na Enfermagem \& Saúde (PARCE\&S) é um programa de ensino de um curso de Enfermagem de uma universidade pública, que tem por objetivo desenvolver ações educativas que tenham significado para todos os sujeitos envolvidos. O referido programa utiliza estratégias de ensino-aprendizagem vinculadas à perspectiva do aprimoramento pessoal, social, profissional e, sobretudo, do aprimoramento e revitalização do conhecimento na Enfermagem e Saúde. Os sujeitos envolvidos nesse programa de ensino são docentes, discentes de Enfermagem, que, interativamente, se propõem a aprender e ensinar de forma contígua, sem sobreposição pedagógica de educadores sobre educandos.

No PARCES\&S, desenvolvem-se ações educativas concernentes às metodologias ativas de aprendizagem, em especial, da aprendizagem significativa. Neste programa, procura-se estimular a interatividade no processo de ensino-aprendizagem e desenvolvê-lo de acordo com as necessidades dos participantes. Enquanto método de ensino e de aprendizagem, optou-se pelos preceitos que regem a aprendizagem significativa, em razão das características e dos objetivos do programa educativo. A razão da escolha da aprendizagem significativa refere-se ao fato de que, dentre as suas acepções, está a valorização de conhecimentos prévios que se relacionam com a estrutura cognitiva do aprendiz. Portanto, coaduna-se como um mecanismo que adquire e contém ideias, assim como informações de distintas áreas do conhecimento ${ }^{1}$.

Ao buscarem a efetividade no processo de ensino-aprendizagem, os educadores apoiam-se em distintas teorias educativas, com destaque para: Behaviorismo, Cognitivismo, Humanismo, Construtivismo. David Ausubel, conjuntamente com Jean Piaget, Jerome Bruner e Joseph Novak são considerados expoentes na linha cognitivista de aprendizagem. A aprendizagem significativa, enquanto concepção educativa, enfatiza o processo de cognição, na qual os indivíduos atribuem significados à realidade em que se encontram, valorizam o processo de compreensão, transformação, armazenamento e utilização das informações advindas do processo de aprendizagem².

$\mathrm{Na}$ aprendizagem significativa, preconiza-se que os educadores precisam criar situações no cotidiano, procurando descobrir o que o indivíduo já sabe. O conhecimento anterior, portanto, é o ponto inicial do processo de aprendizagem, considerando as características dos indivíduos. O foco central da aprendizagem significativa está na identificação dos fatores que efetivam e facilitam a aprendizagem, ou seja, pode ter mais significado, para o discente, a inter-relação entre o novo conceito e a sua estrutura cognitiva, fato que permite ao aprendiz descobrir novos significados, tendo como base seus conhecimentos prévios².

A aprendizagem significativa, portanto, parte da pressuposição de que os novos conhecimentos devam ter significado para os educandos com a valorização do conhecimento prévio dos discentes que, cognitivamente, permite, ao estudante, elaborar novos significados ${ }^{3}$. No fazer educativo, a aprendizagem significativa consubstancia-se em dois princípios: o educando precisa apresentar disposição em aprender, fato que transcende a simples memorização; segundo, o aprendizado, cognitivamente, precisa ter significado ${ }^{3}$.

As metodologias ativas e inovadoras de ensino-aprendizagem estão comprometidas com o desenvolvimento de ações educativas críticas e criativas ${ }^{4}$. Nessas modalidades educativas, os alunos precisam adotar atitudes proativas na aprendizagem, razão da autonomia do processo que permite, ao aluno, transcender a posição passiva, ao acúmulo acrítico e não reflexivo de conteúdos ${ }^{4}$. A utilização de metodologias alternativas consubstancia-se na necessidade de mudar o foco no processo educativo, 
introduzindo acepções pedagógicas ativas de aprender e ensinar, estimulando-se a autonomia, a participação, a criatividade e a responsabilidade do educando 5 .

Nesta concepção educativa, os recursos cognitivos estão relacionados à forma com que os estudantes: estruturam os conteúdos, formam e utilizam diferentes conceitos, interpretam informações, motivações e expectativas que influenciam a aprendizagem. Quando o discente assume a responsabilidade por sua aprendizagem, acaba atribuindo um significado diferenciado ao processo, estando o docente mais no papel de guia, orientador e facilitador do processo de aprendizagem. Para tanto, nesta modalidade, preconiza-se que as ações educativas sejam colocadas em prática, em locais e situações de aprendizagens dinâmicas e significativas, e tenham como base o conhecimento dos educandos ${ }^{6}$. A concepção de autonomia nesse processo pode ser entendida como atitude central do processo educativo, como uma situação na qual os alunos definem seus objetivos de aprendizagem ${ }^{7}$. A autonomia, portanto, implica atitudes ativas, por parte dos discentes, na aquisição de conhecimentos e habilidades, permitindo, aos indivíduos, regularem seu processo educativo ${ }^{7}$. Os indivíduos, ao assimilarem novos conhecimentos, potencializa-os a galgarem mais autonomia no processo educativo ${ }^{2}$.

Para os docentes envolvidos no programa, trabalhar com a aprendizagem significativa permite transcender as metodologias tradicionais. Nesta, assumem o papel de condutores/apoiadores de possibilidade de aprendizagem a partir de situações vivenciadas no trabalho, em especial, a intersecção entre teoria e prática. As práticas educativas, características dessa modalidade, possibilitam, aos sujeitos envolvidos no processo de aprendizagem, a articulação de conhecimentos anteriores interpostos com a construção do novo ${ }^{8}$.

O estudo se justifica na medida em que se entende que a oferta de novos preceitos educativos esteja, também, relacionada à interface entre os diferentes segmentos na construção de novas práticas no campo da saúde. Programas aditivos de ensino podem contribuir na construção de conhecimentos para o desenvolvimento pessoal, social, econômico e cultural dos sujeitos, com vistas à transformação para uma sociedade mais instruída, crítica, responsável, justa, equitativa e tolerante.

Diante do exposto, a questão de pesquisa busca elucidar: Quais são as contribuições da aprendizagem significativa na construção do conhecimento e formação em Enfermagem sob a perspectiva de estudantes de Enfermagem? Portanto, o objetivo foi o de compreender como a aprendizagem significativa pode contribuir para a construção do conhecimento e formação na perspectiva de estudantes de Enfermagem.

\section{Método}

Metodologicamente, o estudo pode ser caracterizado como uma pesquisa qualitativa, descritiva e exploratória ${ }^{9}$. Como critério de inclusão, definiu-se que os sujeitos do estudo deveriam participar do projeto de ensino há, pelo menos, um ano e ter participado de $75 \%$ das atividades. Automaticamente, excluíram-se os sujeitos que não se enquadravam nos critérios. Os sujeitos da pesquisa foram estudantes de Enfermagem que participaram/participam do programa de ensino nos últimos três anos, que, de livre e espontânea vontade, concordaram em participar da pesquisa. Na coleta dos dados, utilizou-se um instrumento com questões semiestruturadas, as quais foram gravadas em sistema digital, e, posteriormente, transcritas. Na apreciação dos dados, empregou-se a proposta de análise temática ${ }^{10}$.

Todos os aspectos éticos foram observados conforme a Resolução 466/2012 ${ }^{11}$, sendo o projeto aprovado por Comitê de Ética em Pesquisa. Em razão da garantia de manutenção do sigilo e anonimato, os sujeitos estão identificados por código que mescla letras e números.

\section{Resultados e discussão}

O programa de ensino denominado PARCE\&S é decorrente da necessidade de se criarem espaços educativos alternativos, dos quais participam, espontaneamente, docentes e discentes, em dias, horários e temas geradores do processo educativo, previamente definidos, com encontros semanais. 
Participam deste grupo, docentes e discentes de um curso de Enfermagem, de uma universidade pública federal do interior do estado do Rio Grande do Sul.

A estratégia educativa utilizada partiu dos preceitos que regem a 'aprendizagem significativa', e os temas a serem trabalhados são definidos pelos participantes do grupo, com programação mensal. Sobre os conteúdos, a recomendação é a de que esses envolvam a intersecção entre a 'clínica e cuidados na Enfermagem'. Cabe, aos discentes, o papel de apresentadores e debatedores dos temas que foram previamente definidos, enquanto os docentes participam fazendo a moderação das discussões, assim como correção de desvios de assuntos e na condução e síntese nos encontros educativos. Este programa de ensino vem sendo desenvolvido há três anos. Paralela e consecutivamente, desenvolveuse um projeto de pesquisa com os participantes do programa de ensino.

Como já referido, os sujeitos do estudo foram doze alunos que regularmente participaram de $75 \%$ do programa de ensino. A análise temática permitiu a construção de duas categorias: Contribuições educativas do PARCE\&S na formação em Enfermagem e Contribuições do PARCE\&S na construção do conhecimento.

\section{Contribuições do PARCE\&S na formação em Enfermagem}

A formação em Enfermagem é complexa, pois envolve múltiplos fatores, necessitando agregar o técnico e o humano, o teórico e o prático, permeada por uma perspectiva crítica e reflexiva pautada em princípios ético-político-filosóficos e qualificada pelo rigor científico na construção do conhecimento. $O$ pensar-agir de forma crítica e reflexiva na formação permite, aos acadêmicos de Enfermagem, um olhar presente mais aguçado das vivências acadêmicas, com as projeções de futuro. Refere um dos sujeitos do estudo que: "Os encontros no PARCE\&S proporcionaram reflexões necessárias para o processo de formação acadêmica, pois, através de estudos e discussões conseguimos associar o embasamento teórico com a rotina do processo de trabalho do profissional enfermeiro"(EE1).

O reconhecimento do programa educativo no desenvolvimento profissional evidencia-se na situação em que a intersecção entre teoria e prática é consubstanciada pelo entendimento de que o conhecimento é coletivamente construído.

“Reconheço o PARCE\&S como instrumento significante na minha formação. Certamente, o conhecimento adquirido durante os períodos de atividades farão diferença na minha atuação profissional, contribuindo no desenvolvimento de técnicas e habilidades, assim como uma reflexão crítica do processo de trabalho". (EE9)

Segundo o entendimento do entrevistado, o uso da aprendizagem significativa no programa educativo, enquanto recurso didático, foi preponderante para a formação crítica e reflexiva e para a construção do conhecimento.

“O PARCE\&S contribuiu positivamente na minha formação acadêmica, já que me permitiu desenvolver temáticas e experiências extracurriculares que foram evidenciadas por mim ou pelos demais integrantes. Por isso, acredito que o projeto tenha me proporcionado uma formação diferenciada, com maior conhecimento, além de ter desenvolvido minhas capacidades, bem como um pensamento mais crítico e reflexivo". (EE10)

A aprendizagem significativa constitui importante recurso didático na formação crítica e reflexiva dos estudantes, nas mais diversas áreas. A utilização dessa estratégia favorece a autonomia do educando, estimula a iniciativa e protagonismo no processo educativo. Para os docentes, a aprendizagem significativa compõe um importante recurso na prática pedagógica como estratégia de inclusão discente na construção do conhecimento ${ }^{12}$.

A utilização de novas estratégias educativas insere o estudante no seu processo de aprendizagem, possibilitando-Ihe: articular teoria e prática, correlacionar conhecimentos, refletir sobre problemas reais 
que envolvem a formação profissional em relação às necessidades do mercado de trabalho, e a sua intersecção e contribuição para o desenvolvimento social, das tecnologias e das ciências ${ }^{13}$. A inclusão de estratégias pedagógicas destinadas à aquisição de conhecimentos com base na realidade favorece a aproximação entre teoria e prática dos futuros profissionais em prol de uma visão crítica de se trabalhar com problemas reais. Portanto, observa-se que o contexto atual requer a formação de enfermeiros ativos, críticos, reflexivos e criativos ${ }^{14}$.

As atividades educativas incluem os discentes, como sujeitos participantes e ativos. Os temas geradores dos encontros são definidos pelos alunos, assim como a condução da atividade educativa. Sobre isso, os participantes afirmam que:

"Eu considero fundamental este projeto na minha formação, uma vez que a metodologia educativa se mostrou muito efetiva, em que todos os acadêmicos se preparavam para as discussões, eram assuntos escolhidos pelos próprios alunos, que sentíamos fragilidade durante a graduação, além disso, em um grupo pequeno as discussões se tornam mais efetivas". (EE2)

"O conhecimento é algo que estará sempre em construção e a oportunidade de participar deste projeto amplificou os meios e formas de aprendizagem. Foram proveitosos nossos estudos nas manhãs de segunda-feira, com toda certeza vai oportunizar outros alunos nessa aprendizagem. Valeu muito". (EE3)

“O projeto PARCE\&S complementa o conhecimento da Enfermagem com métodos alternativos de aprendizagem, estimulando o pensamento do acadêmico para assimilar saberes da clínica e do cuidado em Enfermagem". (EE11)

O processo de aprendizagem não depende tão somente de capacidades de atenção dos alunos em incorporar informações. Necessita-se, também, desenvolver habilidades de análise, processamento e aplicação, e a utilização de estilos e as estratégias de aprendizagem são preponderantes para melhorar o desempenho cognitivo. Os estilos de aprendizagem são susceptíveis de contínuos processos de aprimoramento e qualificação da aprendizagem. O estilo de aprendizado reflexivo caracteriza-se pela ponderação, a consciência, a sensibilidade e a capacidade de análise ${ }^{15}$.

O compromisso com a formação em Enfermagem também está ancorado em estratégias educativas alternativas e envolventes de ensino. Portanto, uma das funções do docente está em: criar possibilidades, estimular a reflexão, orientar os discentes quanto ao seu desenvolvimento, para enfrentar os diferentes desafios, nos mais diversos contextos. Estrategicamente, o docente tem o compromisso de criar e desenvolver condições de ensino com a oferta de modalidades educativas diferenciadas, que remeta, aos estudantes, a responsabilidade da organização e transformação do conhecimento de forma compartilhada ${ }^{6}$.

Especificamente, o uso da aprendizagem significativa na formação em Enfermagem procura articular os conteúdos teóricos com as práticas e as experiências vividas. O método concernente à aprendizagem significativa estimula o discente a ser também protagonista na construção do seu conhecimento, haja vista que a Enfermagem precisa de profissionais habilitados para cuidar de pessoas, com conhecimentos técnicos, teóricos e práticos, balizados pela ética e responsabilidade, entre outros preceitos ${ }^{3}$.

Os extratos de respostas dos entrevistados permitem entender que a participação no programa de ensino contribui na formação em diversos aspectos, sobretudo no que tange às discussões e reflexões, a interação entre a teória e a prática. Reconhece-se que a inserção nesta atividade educativa foi significante para a formação em Enfermagem, razão da gama de conhecimentos que foram agregados. Porquanto, evidencia-se que as contribuições mais importantes referem-se à possibilidade de participar nas discussões, compartilhar conhecimentos e, sobretudo, estimular o pensamento crítico e reflexivo. 


\title{
Contribuições do PARCE\&S na construção do conhecimento
}

A aprendizagem significativa é uma das estratégias de ensino que valoriza conhecimentos prévios e experiências vividas pelos aprendizes. Nesta, o conhecimento anterior é o ponto de partida do processo educativo, considerando as características e necessidades dos indivíduos. Relatam os participantes do estudo que:

\begin{abstract}
"A metodologia utilizada possibilitou uma participação ativa na busca do conhecimento, nas discussões com conteúdos importantes, todos planificados de acordo com o interesse dos componentes do grupo. Em sala de aula, nas disciplinas curriculares muitas vezes não temos abordagens mais completas sobre determinados assuntos e no PARCE\&S nos aprofundávamos no que estava em questão e discussão". (EE3)

“O PARCE\&S contribuiu muito na construção do conhecimento, principalmente pela metodologia aplicada. Estudar os temas abordados e expor aos colegas facilita o aprendizado, além de contribuir na dicção e oratória em público. Esse projeto qualifica os acadêmicos na clínica direcionando as intervenções necessárias e adequadas". (EE6)
\end{abstract}

Entre os desafios do exercício da docência, está o desenvolvimento de estratégias para envolver os estudantes, de forma que os permita inserirem-se ativamente no processo de aprendizagem e serem também ativos no processo educativo e protagonistas na construção do conhecimento. Por sua vez, a aprendizagem significativa permite a exploração de conhecimentos dentro de um determinado contexto, impreterivelmente, valorizando conhecimentos, experiências e envolvendo os estudantes no processo educativo. Nesta estratégia de aprendizado, cabe aos docentes assumirem o papel de construtor de possibilidades de aprendizagem ${ }^{16}$.

A abrangência da aprendizagem significativa, enquanto atividade educativa, busca correlacionar a teoria e prática considerando o estudante como autor na construção do seu conhecimento. A utilização das metodologias ativas de aprendizagem constitui importante adendo na construção do conhecimento do estudante, de forma que os subsidia posteriormente na sua prática profissional ${ }^{17}$.

A necessidade de buscar conhecimentos predeterminados permite a interação e a reflexão coletiva. Sobre o fato, os entrevistados assim se manifestam.

"O PARCE\&S através de sua metodologia de estudo em práticas clínicas contribuiu na construção do conhecimento durante todo o seu período de atividades". (EE9)

"A metodologia educativa utilizada no grupo me estimulou a pesquisar, ler, buscar, aprender sobre a temática a ser discutida nos próximos encontros, utilizando exclusivamente informações nas fontes baseadas em evidências científicas, o que acredito ter me proporcionado um conhecimento mais consistente e fidedigno, além de ser aplicável na prática do cuidado em saúde. Outro aspecto válido na metodologia utilizada refere-se às discussões, na qual procurávamos sanar dúvidas nos textos que havíamos utilizado". (EE10)

Novas modalidades educativas permitem, ao estudante, a participação ativa no seu processo de aprendizagem, buscando conhecimentos, articulando teoria e prática, correlacionando saberes e fazendo reflexões críticas sobre problemas reais que inferem na sua formação e posterior exercício profissional, com vistas a contribuir para o desenvolvimento social e de novas tecnologias. Nas atividades educativas, que se utilizam dos preceitos que regem a aprendizagem significativa, a participação dos discentes é fundamental. Nesta prática educativa, o estudante transcende a postura de receptor do processo, e a reconfiguração do seu papel no processo educativo, transcendendo a uma posição passiva no processo educativo, assumindo participação ativa na construção do conhecimento ${ }^{13}$.

O significado e a importância do PARCE\&S na construção do conhecimento e na complementaridade do processo educativo são descritos como: 
“Foi muito significativa a importância do PARCE\&S na minha construção de conhecimento, pois assuntos de clínica muitas vezes são falados rapidamente em sala de aula. Muitos dos assuntos que discutimos no projeto estão presentes no dia a dia, mas a importância do PARCE\&S não se limita a isso, pois me direcionou a construir um olhar voltado às questões clínicas, avaliação do paciente, correlacionar e compreender além do que está posto aos olhos". (EE2)

“O PARCE\&S foi uma grande experiência sob o ponto de vista do aprimoramento do conhecimento. Por tratar-se de um espaço em que eram abordadas questões clínicas da atuação do enfermeiro, foi possível instigar-nos a buscar mais sobre os temas pré-definidos, discutir sobre as condutas mais adequadas e, consequentemente desenvolver um raciocínio mais clínico". (EE4)

O desenvolvimento de novas estratégias pedagógicas está atrelado a um processo educativo que transcende a formação técnica, razão que ancora em preceitos educativos críticos e transformadores, que, contextualmente, contribuem para a conscientização dos sujeitos, para uma prática social emancipatória e uma sociedade justa e sustentável ${ }^{13}$.

$\mathrm{Na}$ construção do conhecimento, os preceitos educativos precisam estar articulados com as necessidades dos estudantes, razão por que as situações e problemas que se interpõem no processo educativo buscam despertar o interesse dos aprendizes, correlacionando o aprendizado ao conhecimento prévio dos discentes. Associar o pensar e o fazer, refletir o teórico e o prático, instigar o questionamento e a controvérsia, são alternativas de superação aos modelos baseados na transmissão vertical de conhecimentos ${ }^{14}$.

A participação do estudante é fundamental na construção do conhecimento, haja vista o comprometimento na escolha dos temas geradores da ação educativa, assim como sua inserção e participação no processo:

"A participação no projeto foi de grande importância para a construção de meu conhecimento, levando em conta a dinâmica utilizada para os encontros, onde eram discutidos, abordados e repensados diversos assuntos de interesse comum entre o grupo. Os assuntos eram escolhidos pelos próprios participantes, o que aproxima e faz com que o reconhecimento e apreensão do tema abordado se dêem de forma mais efetiva e qualificada". (EE5)

"O PARCE\&S foi de suma importância na construção do conhecimento pessoal que reflete no aprimoramento profissional, uma vez que o estudo e consequente discussão em grupo facilitam a compreensão dos assuntos abordados, sendo de forma dinâmica e informal". (EE7)

$\mathrm{Na}$ construção do conhecimento, aprender significativamente implica agregar novas perspectivas ao conhecimento. Na Enfermagem, em especial, o conhecimento na resolução de casos clínicos com situações características do cotidiano, permite despertar o raciocínio crítico dos estudantes e consubstanciar a tomada de decisões. Consubstancia-se que a utilização de metodologias ativas de ensino/aprendizagem seja uma das formas mais profícuas para estimular o pensamento crítico dos estudantes ${ }^{2}$. Atividades que envolvem projetos educativos contribuem na aproximação de teoria e prática, facilitam o senso crítico do estudante ${ }^{8}$.

$\mathrm{Na}$ aprendizagem significativa o conhecimento prévio é base para a incorporação, compreensão e fixação de novos conhecimentos ${ }^{2}$. Na construção do conhecimento, o uso das metodologias ativas de aprendizagem constitui importante recurso pedagógico para a formação crítica e reflexiva. $\mathrm{Na}$ perspectiva da construção do conhecimento, carece ao estudante redimensionar o seu papel no processo educativo e transcender a atitudes passivas e receptivas de informações, para assumir uma posição ativa e participativa no processo de construção do conhecimento ${ }^{13}$.

Para os participantes do estudo, a inserção no programa contribui em inúmeros aspectos, razão por que possibilitou: discussões e interações; o aprofundamento de disciplinas curriculares; superação de fragilidades na intersecção de teoria e prática; a possibilidade de superar limitações, como a 
introversão e a insegurança de dialogar em grupos. Por conseguinte, evidencia-se, claramente, que a principal contribuição refere-se à construção e ao aprimoramento do conhecimento.

\section{Conclusão}

O objetivo do programa foi desenvolver ações educativas que estivessem consignadas com as necessidades e/ou realidades dos sujeitos envolvidos no processo educativo; proporcionar aos sujeitos a possibilidade de participarem ativamente do seu processo educativo; contribuir para o aprimoramento e qualificação da atenção e da gestão em saúde.

A oferta de diferentes estratégias educativas contribui para a formação em Enfermagem, razão pela qual se utiliza de formas diferenciadas de aprender e ensinar. Nesta modalidade educativa, os discentes precisam repensar e reconfigurar sua atuação no processo educativo, saindo de uma posição passiva, e adotar atitudes ativas, sendo o responsável pela definição e seleção dos conteúdos, pela apresentação e debates e, também, por desenvolver a capacidade de sustentar academicamente preceitos teóricos nas mais diferentes áreas do conhecimento.

A modalidade educativa, ora vivenciada, é uma opção metodológica plenamente viável de superação à crítica na construção do conhecimento, de ações educativas verticalizadas com docentes exercendo o papel de reprodutor de conteúdos, estando o estudante em uma posição passiva de expectador, com possibilidades diminutas de participar, interagir, refletir e adotar condutas, até porque pode não haver estímulos a este tipo de atitude.

Ações educativas com base nas metodologias educativas de aprendizagem ainda encontram resistências quanto a sua implantação. Na realidade vivida, vem se trabalhando com esses preceitos educativos em disciplinas optativas e complementares de graduação e, também, com grupos menores de estudantes que participam de programas, projetos, práticas e estágios. O PARCE\&S reúne, em média, 10-15 alunos, e tem como maior entrave a coincidência de horários das aulas, práticas e dos estágios. Como traduzido pelas respostas dos sujeitos do estudo, evidencia-se que programas desta natureza contribuem significativamente para a construção do conhecimento e formação em Enfermagem. Por fim, reconhece-se a limitação do estudo, fato que nos instiga a olhar com mais propriedade o contexto vivido e projetar novas pesquisas sobre a temática.

\section{Colaboradores}

Luiz Anildo Anacleto da Silva foi responsável pela análise dos dados e redação textual. Rafael Marcelo Soder e Cínthia Cristina Oliveski colaboraram na redação textual. Mirian Natale Frizzo colaborou na coleta de dados e na redação textual. 


\section{Referências}

1. Moreira MA. Aprendizagem significativa: um conceito subjacente. Aprendiz Significativa Rev. 2013; 1(3):25-46.

2. Distler RR. Contribuições de David Ausubel para a intervenção psicopedagógica. Rev Psicopedag. 2015; 32(98):191-9.

3. Sousa ATO, Formiga NS, Oliveira SHS, Costa MML, Soares MJGO. A utilização da teoria da aprendizagem significativa no ensino da enfermagem. Rev Bras Enferm. 2015; 68(4):713-22.

4. Cotta RMM, Costa GD, Mendonça ÉT. Portfólio reflexivo: uma proposta de ensino e aprendizagem orientada por competências. Cienc Saude Colet. 2013; 18(6):1847-56.

5. Cardoso DSA, Oliveira JM, Costa LMC, Rozendo CA. Aprendizagem reflexiva: o uso do portfólio coletivo. Rev Bras Educ Med. 2015; 39(3):442-9.

6. Fernández V, Beligoy M. Estilos de aprendizaje y su relación con la necesidad de reestructuración de las estrategias de aprendizaje de los estudiantes universitarios de primer año. FEM. 2015; 18(5):361-6.

7. González-Hernando C, Carbonero-Martín MÁ, Lara-Ortega F, Martín-Villamor P.

"Learning to learn" in nursing higher education. Invest Educ Enferm. 2012; 31(3):473-9.

8. Carvalho PSRP, Rego ALC, Ferreira KS, Silva SB, Vitor AF, Ferreira JMF. Teoria da aprendizagem significativa como proposta para inovação no ensino de enfermagem: experiência dos estudantes. Rev Enferm UFSM. 2015; 5(1):186-92.

9. Gil AC. Como elaborar projetos de pesquisa. 5a ed. São Paulo: Atlas; 2010.

10. Bardin L. Análise de conteúdo. São Paulo: Edições 70; 2011.

11. Ministério da Saúde (BR). Resolução n 466, de 12 de dezembro de 2012. Brasília, DF: 2012 [citado 27 Maio 2016]. Disponível em: http://conselho.saude.gov.br/ resolucoes/2012/Reso466.pdf.

12. Borges TS, Alencar G. Metodologias ativas na promoção da formação crítica do estudante: o uso das metodologias ativas como recurso didático na formação crítica do estudante do Ensino Superior. Cairu Rev. 2014; 3(4):119-43.

13. Gemignani EYMY. Formação de professores e metodologias ativas de ensinoaprendizagem: ensinar para a compreensão. Rev Front Educ. 2012; 1(2):1-27.

14. Mesquita SKC, Meneses RMV, Ramos DKR. Metodologias ativas de ensino/ aprendizagem: dificuldades de docentes de um curso de enfermagem. Trab Educ Saude. 2016; 14(2):473-86.

15. Bahamón Muñetón MJ, Vianchá Pinzón MA, Alarcón Alarcón LL, Bohórquez Olaya $\mathrm{Cl}$. Estilos y estrategias de aprendizaje relacionados con el logro académico en estudiantes universitarios. Pensam Psicol. 2013; 11(1):115-29.

16. Kalinowski CE, Massoquetti RMD, Peres AM, Larocca LM, Cunha ICKO, Gonçalves LS, et al. Metodologias participativas no ensino da administração em enfermagem. Interface (Botucatu). 2013; 17(47):959-67.

17. Limberger JB. Metodologias ativas de ensino-aprendizagem para educação farmacêutica: um relato de experiência. Interface (Botucatu). 2013; 17(47):969-75. 
Silva LAA, Soder RM, Oliveski CC, Frizzo MN. Programa de Mejoramiento y Revitalización del Conocimiento en Enfermería y Salud. Interface (Botucatu). 2018; 22(65):517-26.

El objetivo del artículo es entender como el aprendizaje significativo puede contribuir a la construcción del conocimiento y la formación en la perspectiva de los estudiantes de Enfermería. Se trata de una investigación cualitativa, descriptiva y exploratoria. Los sujetos fueron 12 estudiantes de un curso de Enfermería y participantes en un programa de enseñanza. Los resultados permitieron la construcción de dos categorías: contribuciones en la formación y en la construcción del conocimiento. Llegamos a la conclusión de que la provisión de programas educativos con metodologías alternativas contribuí en la construcción del conocimiento y formación en Enfermería. Uno de los principales factores contribuyentes se refiere a la posibilidad de construcción colectiva, proporcionando a los estudiantes una participación activa, crítica y reflexiva en las actividades educativas.

Palabras clave: Enfermería. Educación en Enfermería. Estudiantes de Enfermería. 